\title{
Benign Metastasizing Leiomyoma and Intravenous Leiomyomatosis Following Total Hysterectomy: Two Case Reports and a Literature Review
}

\author{
Nathaniel A Parker DO ${ }^{1 *}$, Christopher SR Dakhil MD², Shaker R Dakhil MD² and Daniel Lalich MD ${ }^{3}$ \\ ${ }^{1}$ Resident Physician, University of Kansas School of Medicine Wichita, Wichita, USA \\ ${ }^{2}$ Medical Oncologist, Cancer Center of Kansas, Wichita, USA \\ ${ }^{3}$ Department of Pathology, Wesley Medical Center, Wichita, USA
}

Received: 琒July 11, 2018; Published: 眥July 23, 2018

*Corresponding author: Nathaniel A Parker, Resident Physician, University of Kansas School of Medicine-Wichita, Wichita, USA

\begin{abstract}
Uterine leiomyomas are the most common gynecological tumor in women of reproductive age. These tumors are typically benign entities, but uncommonly transition toward tissues of malignant potential. Rare growth patterns of uterine leiomyomata have been observed which include benign metastasizing leiomyoma (BML), disseminated peritoneal leiomyomatosis (DPL), intravenous leiomyomatosis (IVL), retroperitoneal leiomyomatosis (RPL) and parasitic leiomyoma (PL). Metastasis of uterine fibroids most commonly appear several years after the diagnosis and removal of uterine leiomyomata by hysterectomy. For two women who sought treatment for BML and IVL in our clinic, we retrospectively outlined and evaluated their specific genetic, pathological, and clinical features with the intention to elucidate possible treatment options.
\end{abstract}

\section{Introduction}

Uterine leiomyomas have been shown to affect up to $30 \%$ of women of reproductive ages. They represent the most common gynecologic neoplasm in women [1,2]. Diagnosis of classic uterine leiomyomata by radiology is historically not complex given their typical features on imaging and clinical manifestations. Leiomyomas most commonly arise from the uterus, but can uncharacteristically originate in the vulva, ovaries, bladder, and urethra2. In addition, on rare occasions they have been discovered in the tissues of the skin, soft tissues, skeletal muscle, bone, lymph nodes, mesentery, and retroperitoneum [3]. Benign metastasizing leiomyoma (BML) is a rare entity. The term itself represents a contradiction in nomenclature. BML can be observed as a mass with histologically benign features, but can also demonstrate metastatic potential and present with diffuse lung tumors. First described by Steiner, Marshall, and Morris, this rare disease has gone by many titles. Steiner recommended the use of the term "metastasizing fibroleiomyoma," as he thought the label of "benign" was incorrect. BML has also been called leiomyomatous hamartoma and pulmonary chondroid Mesenchymoma [4-6]. BML is predominantly

diagnosed incidentally as patients are usually asymptomatic at the time of tumor discovery [7]. If symptoms are present, they most commonly include shortness of breath, cough, and/or chest pain. In BML specifically, the lungs are the most common site of metastasis. Patients with BML commonly have an indolent clinical course and favorable outcome. In the review of 10 cases of BML, only one patient died from BML-related complications [8]. The etiology of BML is not completely understood. Numerous hypotheses have been proposed, but currently vascular dissemination appears to be the most widely accepted [4-6]. Most women with BML have a past medical history of therapeutic hysterectomy or myomectomy for uterine fibroids. Thus, some researchers hypothesize prior surgical events induce vascular spread. Interestingly, certain cases of BML have been diagnosed concomitantly with primary uterine leiomyoma or in unusual locations supporting a theory of multifocal origin [9-12].

Intravenous leiomyomatosis (IVL) is historically less common than BML. Even more of an oddity, IVL can extend into the cardiopulmonary system including the right atrium, right ventricle, 
and pulmonary arteries. When this manifestation of IVL occurs, IVL is then termed intracardiac leiomyomatosis (ICLM). ICLM was first described by non-English texts in the early 1900s [13]. In 1974, ICLM were first reported in English [14]. Likely due to technological advancements in imaging techniques, ICLM is being reported more often. However, definitive diagnosis is ultimately determined by postoperative pathological evaluation. Based on a 2012 comprehensive analysis of electronic literature databases MEDLINE/PubMed, Web of Science, and Cochrane Library, ICLM may be reported more frequently than previously thought [15]. However, it remains difficult to ascertain if ICLM's current frequency is more common than BML, or any of the remaining atypical growth patterns of the uterine leiomyomas. Review of the literature and previously published comprehensive searches, we found 118 articles describing ICLM. Screening criteria included English publications that characterized original ICLM discovery by pathological, procedural, or imaging modalities. Articles characterizing ICLM further after original diagnosis, second-stage surgical resections, recurrence, follow-up, or post-operative management were not included. ICLM was found to be solely observed in females. Li et al.'s comprehensive analysis using broader search and screening criteria, determined ICLM that affect women with an average age of 47.6 years, had prior hysterectomy ( $>50 \%$ ), and concomitantly diagnosed with uterine leiomyoma $(\sim 30 \%)$. Symptoms at the time of initial presentation and ICLM diagnosis are non-specific. Thus, other cardiac tumors should remain on differential diagnosis list until definitive diagnosis by pathology is performed. Li et al noted approximately $80 \%$ of ICLM cases to originate from either the iliac or ovarian veins and terminate into right atrium and ventricle.
ICLM is histologically benign. Thus, necrosis, mitoses, or cellular irregularities are rare [15].

\section{Case Reports}

\section{Case 1}

A non-smoking 30-year-old Vietnamese woman with a cancernegative family history was referred for lung lesions. Two years prior, she underwent total hysterectomy. At that time, a single uterine fibroid was noted and excised. Pathology confirmed a diagnosis of leiomyoma. At the time of presentation, patient complained of only flu-like symptoms. Chest x-rays showed bilateral lung lesions. Subsequently an extensive work up resulted in unremarkable CT scans, bronchoscopy, and thoracoscopy. Lung tissue biopsy showed benign-appearing smooth muscle nodules, suggestive of BML. Over the next two decades, patient's lung lesions persisted despite proposed therapeutic regiments by per MD. Anderson Cancer Center that included megestrol, tamoxifen, medroxyprogesterone, leuprolide and letrozole. No curative therapy was identified. Nineyears post-hysterectomy, additional lung lesion tissue acquired. Immunostaining revealed full negativity to c-kit and Her2/neu, but mixed negative EGFR results with slightly positive staining of material among muscle cells and not the muscle cells themselves. Chemoradiation was discussed but not administered based on patient's minimal symptomatology following previous exhaustive treatment regiments. Recent, genomic sequencing by Foundation testing was performed by blood samples. An ALK: N1532D genomic variant was discovered. Currently, patient is being conservatively managed with observation and symptom management

\section{Case 2}

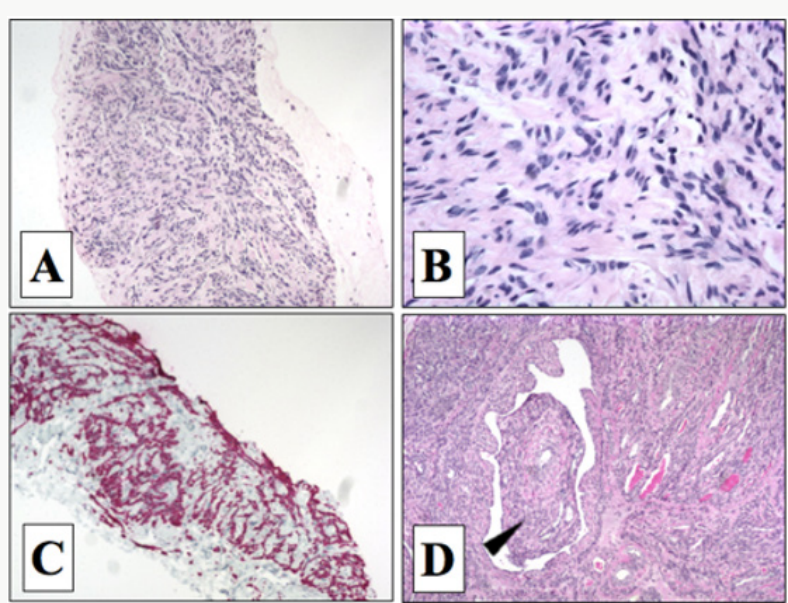

Figure 1: Histologic section of adnexal mass from 33-year-old female (Case 2) with suspected metastatic leiomyomatosis.

A. Photomicrograph of adnexal mass tissue extending to right ovary and cross-sectioned ( $\mathrm{H}$ and E x10)

B. Photomicrograph of same section as A, but at high magnification ( $\mathrm{H}$ and $\mathrm{E} \times 40)$.

C. Histologic section stained to detect tissues of a Mullerian origin. Photomicrograph at $x 10$ showing red-pink coloration denoting positive immunohistochemical staining of spindle cells appearing to be smooth muscle in nature for desmin.

D. Histologic section determined to originate from benign uterine fibroid ( $\mathrm{H}$ and $\mathrm{E} \times 40$ ). Note the predominate leiomyomatous tissue in entire cross-section possessing a vascular component within adnexal blood vessel to form an intravascular thrombus (arrowhead). These observations, together with tissue's benign features, but also unique appendage-like vascular configurations, support intravenous leiomyomatosis being the primary pathogenic culprit. 
A Caucasian 33-year-old woman of European descent presented with abdominal pain. CT scans showed an adnexal mass. Total hysterectomy and bilateral oophorectomy were performed. At that time, a well-circumscribed mass was noted. Histopathology supported diagnosis of leiomyoma due to presence of spindle cells without cytologic atypia, low mitotic activity, and without malignant features (Figures 1A \& 1B). (Figure 1C) Over the course of ten years post-hysterectomy, patient developed lung, cardiac, and intra-abdominal masses. Subsequent exploratory procedures revealed further gynecological tissue involvement, as well as retroperitoneal and extensive caval thrombus that extending bidirectionally into iliac veins and right atrium (Figure 1D). Urgent therapy by thrombus and mass removal, IVC reconstruction, tricuspid valve replacement and nephrectomy was performed. Tumor tissue sampling performed at all affected regions. Pathology revealed tumors made of spindle cells without necrosis and mitotic events. Immunostains were negative for CD10, sesmin, anti-SM, inhibin, S100, CD17, pancytokeratin CK7, and RCC. Tissues with a vascular background were positive for an additional marker, CD34 (e.g. right atrium). Desmin, actin, estrogen receptor, and WT-1 reacted positively (Figure 1C) supporting a Mullerian origin. Thus, IVL determined to be most likely etiology considering patient's clinical course, tumor progression, and histopathological findings. After the patient's complex surgical interventions, she was discharged and continued to be followed as an outpatient. Recently, the patient's tumor tissue specimens were analyzed with the genomic sequencing assay Foundation. Testing identified three variants of unknown significance - EGFR: V674I, ERBB4:K1002R, and TSC2:L826M.

\section{Discussion}

\section{Pathogenesis \& Uncommon Growth Patterns}

Metastasis of uterine fibroids most commonly appear several years after the diagnosis and removal of uterine leiomyomata by total hysterectomy. In our two cases the interval between uterine fibroid diagnosis, hysterectomy, and metastasis was two and zero years, respectively. An earlier report reviewing ten cases of BML observed an interval range of four to 23 years (mean 14.9 years) from the time of hysterectomy to BML diagnosis [8]. The data remained consistent with preceding case reports that observed the interval between hysterectomy to BML diagnosis that ranged from three to 20 years (mean 10 years) [5]. Uterine leiomyomas are the most common gynecological tumor in women of reproductive age.
Prevalence has been found to be approximately 50\% in women > 30 years of age $[1,2]$. Far less common are the rare growth patterns of uterine leiomyomata: benign metastasizing leiomyoma (BML), disseminated peritoneal leiomyomatosis (DPL), intravenous leiomyomatosis (IVL), retroperitoneal leiomyomatosis (RPL), and parasitic leiomyoma (PL) (Table 1). These growth patterns have been documented and considered benign. Certain benign neoplasms have the potential to be precursors to malignant transformation. Colonic villous adenomas are a classic example. If left untreated, they frequently develop into malignant cancer [16]. However, most benign tumors rarely go through a similar progression (e.g. uterine leiomyomas, pleomorphic adenoma), and some almost never (e.g. lipomas) [17]. Thus, experts have determined that benign tumors have significantly lower malignancy potential. Earlier investigations have determined a low mitotic index ( $<5$ mitoses / 10 high power field, 400X, $2.7 \mathrm{~mm}^{2}$ ) and the absence of necrosis and atypia are the most helpful pathologic features that characterize BML [18]. The pathogenesis of each atypical growth pattern of uterine leiomyoma is controversial. They have been found to be the result of clonal expansion of smooth muscle cells of the uterus, without significant cellular atypia or high mitotic index $[19,20]$. In contrast, leiomyosarcomas frequently exhibit the higher turnover rates and atypia, but overall are seen rarely with frequency rates of 0.1 to $6 \%$ [21]. Certain investigators suggest that primary lesions could be low-grade, slow growing leiomyosarcomas with inherently intact metastatic potential [8]. Also, it's been postulated erroneous sampling could falsely support the diagnosis of benignity [12]. However, recent cytogenetic studies have refuted this claim by showing that in contrast to leiomyosarcomas, BML lesions have identical X-chromosome inactivation and a balanced karyotype $[22,23]$. Canzonieri et al. were the first to describe in detail the relationship between BML, uterine leiomyomas with vascular invasion (LWVI), and IVL. They ultimately suggested not only BML's is equivalency to IWVI, but also the entities simply represent different snapshots on the same timeline. They postulated IVL may represent the eventual metastatic component of LWVI or BML, thus supporting both as potential precursors to IVL. It was believed in certain situations LWVI could be the antecedent to both BML or IVL [24]. Some authors have denied any relationships between the separate pathogenic origins [25]. Others have stated cases of BML with the characteristically absent histological evidence of blood vessel invasion could be explained by insufficient primary tumor sampling [26].

Table 1: Atypical growth patterns of the uterine leiomyoma $2,47-50$

\begin{tabular}{|c|c|c|c|c|}
\hline & Presentation & Differential Diagnosis & Findings (pathological,clinical) & Pathogenesis \\
\hline \multirow{5}{*}{ BML } & - indolent & - malignant lung metastasis & $\begin{array}{l}\text { solitary to multiple subcentimetric lung } \\
\text { nodules }\end{array}$ & hematogenous spread \\
\hline & - dyspnea, chest pain & - infectious granuloma & - cavitary lung lesions & independent multiple foci \\
\hline & - respiratory distress & - sarcoid & - cavitary lung lesions & - hormone driven \\
\hline & & - rheumatoid nodules & - concomitant uterine leiomyoma, DPL, or IVL & \\
\hline & & - amyloidosis & & \\
\hline
\end{tabular}




\begin{tabular}{|c|c|c|c|c|}
\hline \multirow{5}{*}{ DPL } & - indolent & - peritoneal carcinomatosis & - multiple peritoneal masses & - mesenchymal metaplasia \\
\hline & & - mesothelioma & - abdominopelvic nodules & - trauma/surgery-mediated \\
\hline & & - lymphoma & - prior endometriosis & - hormone driven \\
\hline & & - tuberculosis & & \\
\hline & & - desmoid tumor & & \\
\hline \multirow{4}{*}{ IVL } & - aggressive & - caval leiomyosarcoma & $\begin{array}{l}\text { - uterine and/or systemic vein intraluminal } \\
\text { growth }\end{array}$ & $\begin{array}{l}\text { - residual implants or } \\
\text { coexistent uterine fibroids }\end{array}$ \\
\hline & - progressive dyspnea & - right atrial myxoma & - cordlike vessel lesions & \\
\hline & - thrombus & - cordlike vessel lesions & & \\
\hline & $\begin{array}{c}\text { - cardiac metastasis via } \\
\text { RCC, Wilms tumor, or other } \\
\text { neoplasm }\end{array}$ & $\begin{array}{l}\text { - intracardiac, tricuspid } \\
\text { valve insufficiency }\end{array}$ & & \\
\hline \multirow{5}{*}{ RPL } & - indolent & - sarcomas & - concomitant uterine leiomyoma (40\%) & $\begin{array}{l}\text { - residual implants or } \\
\text { coexistent uterine fibroids }\end{array}$ \\
\hline & - back pain & - neurogenic neoplasms & - commonly below renal hilum level & - hormone driven \\
\hline & & - teratoma & & \\
\hline & & - hemangioma & & \\
\hline & & - lymphoma & & \\
\hline \multirow{3}{*}{ PL } & $\begin{array}{l}\text { - urinary outflow } \\
\text { obstruction }\end{array}$ & - pelvic and ovarian masses & - myxoid or hyaline degeneration & $\begin{array}{l}\text { - loss of uterine } \\
\text { attachments, subsequent } \\
\text { new adherence }\end{array}$ \\
\hline & - hydronephrosis & - broad ligament cysts & - Meigs syndrome & - hormone driven \\
\hline & & - lymphadenopathy & - elevated CA-125 & \\
\hline
\end{tabular}

BML, benign metastasizing leiomyoma; DPL, disseminated peritoneal leiomyomatosis; IVL, intravenous leiomyomatosis; RPL, retroperitoneal eiomyomatosis; PL, parasitic leiomyoma

\section{Genomic Sequencing}

Table 2: Established and predicted implications of variants of unknown significance (VUS).

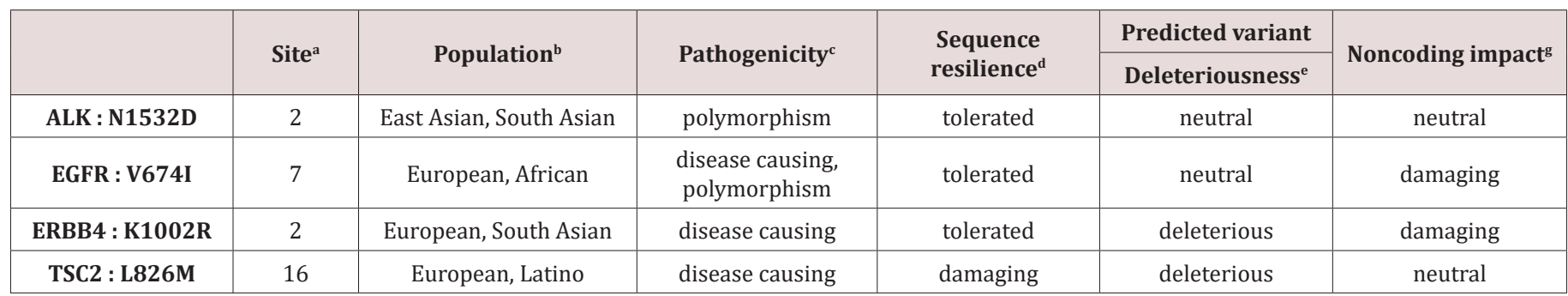

${ }^{\mathrm{a}}$ Chromosome location

${ }^{\text {b}}$ Two most common sub-populations with highest population frequency of alternate alleles.

'Disease potential output of missense variation determined to be either 'disease causing' or 'polymorphism' or both. In silico prediction tool for variant pathogenicity.

${ }^{\mathrm{d}}$ In silico Functional Analysis through Hidden Markov Models (FATHMM) predicting effects of missense mutations on overall tolerance of conserved protein domains. Based on sequence conservation and relative amounts of disease-associated and functionally neutral amino acids in conserved protein regions.

eLikelihood ratio test (LRT) predicts deleterious variants through identification of highly conserved amino acid regions using a comparative genomics data set of 32 vertebrate species.

${ }^{\mathrm{f}} \mathrm{dbNSFP}$ database mutation assessor prediction of variation impact on protein function through sequence conservation. Variant predictions have been trained on COSMIC database and validated (MutationAssesor).

gFATHMM-MKL predicts noncoding effects by integrating functional annotation information. [27-31].

Selected samples from the case patients were subjected to genomic sequencing. Genomic sequencing analysis did not detect any of these mutations in either patient. However, one variant of unknown significance (VUS) was detected in Case 1 - ALK: N1532D, and three were detected in Case 2 - EGFR: V674I; ERBB4:K1002R; and TSC2:L826M. Microsatellite status (MS) was determined to be stable, and the overall tumor mutation burden (TMB) was low (0.80 mutations/Mb) in both patient's genomic sequencing 
analysis. Open source variant databases detailing their possible pathogenicity based on evolutionary conservation, splice-site, mRNA, protein, and regulatory features is described in (Table 2). As there is only limited data available for the relatively small minority of variants, reliance must be placed on computerized prediction tools ("in silica") to help determine whether a variant is damaging or benign. All variants were found to be missense single nucleotide polymorphisms (SNP) in exonic coding regions. However, overall tolerance to genomic alterations in the specific coding region was high. Also, recent data predicts minimal deleterious effects for most variants discovered in our case patients, as well as a sufficient conservation of normal protein function. These predictions of site-specific variations being well tolerated could possible be due to regional genomic changes being common for that area [27-31]. Interestingly, anaplastic lymphoma receptor tyrosine kinase (ALK) gene has been shown to be altered in pediatric neuroblastoma tumors, adult thyroid carcinomas, and metastatic nonsmall cell lung carcinoma [32]. Somatically altered epidermal growth factor receptor (EGFR, ErbB1, HER1) has been observed in a number of epithelial-derived squamous cell carcinomas (e.g. lung, head/neck, anal), most notably in alveolar lung tissue [33]. Mutations in EGFR have been known to result in uncontrolled cellular proliferation. Heightened VUS awareness poses a challenge to physicians not only for determining their relevancy, but for effectively communicating their importance to patients. VUS are termed as such due to: 1.) inadequate characterization in the literature; and/or 2) the genetic alteration found has unclear significance [34]. The complete functional effect of most variants remains unclear, especially when it involves missense mutations. Recent efforts have been made by National Center for Biotechnology Information (NCBI) to track and catalogue newly discovered variants with clinically relevant phenotypes. The NCBI database notes newly discovered variants with clinically relevant phenotypes [27-34].

\section{Treatment}

The benign versus malignant potential of BML and IVL remains unclear. Currently, there are no definitive guidelines regarding management. Earlier reports show BML is not only reliant on estrogen and progesterone, but also the majority BML tumors are ER positive [35]. In postmenopausal women, lung nodules will stabilize or regress. Also, spontaneous regression of metastatic BML lesions has been observed during low estrogen states (e.g. pregnancy, abortion, post-menopause) [36]. In contrast, premenopausal women often experience disease worsening [37]. Thus, bilateral oophorectomy has reportedly been used in previous cases to inhibit tumor progression [38,39]. GnRH analogs have been successful in treating BML [40,41]. Progesterone antagonists have been discussed as possible adjuvant therapy for BML patients, but certain investigators advise against the use of these agents, at least not alone, because of their ability to up-regulate estrogen receptors [42]. Anastrozole's effects have been found to be augmented if paired with a SERM (e.g. raloxifene). Anastrozole inhibits aromatase-P450 enzyme. Uterine leiomyomas have increased amounts of aromatase-P450, which may owe to increased capabilities to penetrate into adjacent myometrium [43-45]. Rivera et al. believed anastrozole and raloxifene combination therapy could be as effective as the more traditionally used GnRH agonists and progesterone, even in postmenopausal patients with BML [46]. Based on BML's close relationship to uterine leiomyomas, some investigators are optimistic raloxifene could be a suitable treatment option for BML. The literature is unclear on the effectiveness of tamoxifen on BML lesions. Rivera et al. reported a short course of daily tamoxifen worsened symptoms in a 47 year-old woman with BML of unknown menopausal status. Saynajakangas and colleagues studying tamoxifen use for the treatment of BML found no significant change in pulmonary nodule size [40].

ICLM is histologically benign. However, ICLM is suggested to be clinically aggressive due to the risk of sudden death caused by total outflow tract obstruction. Complete removal is the recommended treatment. Neoadjuvant and adjuvant anti-estrogen regiments or radiation therapy alone have not been shown to be a curative solution. This is due to the historical nature of IVL tumors to be incompletely hormone-driven. Finally, incomplete removal is not recommended due to previous studies reporting a near $30 \%$ recurrence rate if complete removal is not performed [15].

\section{Conclusion}

Uterine leiomyomas are the most common gynecological tumor in women of reproductive age. These tumors are typically benign entities, but uncommonly transition toward tissues of malignant potential. Rare growth patterns of uterine leiomyomata have been observed which include BML, DPL, IVL, RPL, and PL. Metastasis of uterine fibroids most commonly appear several years after the diagnosis and removal of uterine leiomyomata by hysterectomy [47]. For two women who sought treatment for BML and IVL in our clinic, we retrospectively outlined and evaluated their specific genetic, pathological, and clinical features with the intention to elucidate possible treatment options. Currently, there are no definitive guidelines regarding the management of BML or IVL. Due to BML being typically hormone-driven, a patient's inherent state of low estrogen and/or age must remain an important factor [48]. Thus, hormone therapies by way of receptor and/or feedback pathway inhibition are the primary treatment options for individuals with BML. However, the success of these therapies has been inconsistent based on literature. In contrast, IVL with cardiac extension is not typically hormone-driven. Thus, surgical intervention by complete removal is recommended. Genomic sequencing by Foundation testing of blood and tissue samples from the patients in Case 1 and 2 identified no genomic alterations in any currently established cancer-related gene. However, VUS were detected in both samples from each case. These variants are termed as such because their alterations may have not been adequately characterized in the scientific literature at the time genomic sequencing was performed and/or the genomic context of these variants remains unclear. Thus, their clinical significance can neither be supported, nor 
denied $[49,50]$. We postulate that these variants could possibly be targeted as treatment options in the future when patients have failed all other previous therapies.

\section{Acknowledgement}

Informed, written permission from the subjects of this Case Report is on file with the Cancer Center of Kansas and the corresponding author.

\section{References}

1. Buttram VC Jr, RC Reiter (1981) Uterine leiomyomata: etiology, symptomatology, and management. Fertil Steril 36(4): 433-445.

2. Fasih N, Prasad Shanbhogue AK, Macdonald DB, Fraser-Hill MA, Papadatos D, et al. (2008) Leiomyomas beyond the uterus: unusual locations, rare manifestations. Radiographics 28(7): 1931-1948.

3. Lim SY, Park JC, Bae JG, Kim JI, Rhee JH, et al. (2011) Pulmonary and retroperitoneal benign metastasizing leiomyoma. Clin Exp Reprod Med 38(3): 174-177.

4. Marshall JF, DS Morris (1959) Intravenous leiomyomatosis of the uterus and pelvis: case report. Ann Surg 149(1): 126-134.

5. Steiner PE (1939) Metastasizing fibroleiomyoma of the uterus: Report of a case and review of the literature. Am J Pathol 15(1): 89-110.

6. Van den Bosch JM, SS Wagenaar, B Corrin, JR Elbers, PJ Knaepen, et al. (1987) Mesenchymoma of the lung (so called hamartoma): a review of 154 parenchymal and endobronchial cases. Thorax 42(10): 790-793.

7. Jautzke G, E Muller Ruchholtz, U Thalmann (1996) Immunohistological detection of estrogen and progesterone receptors in multiple and well differentiated leiomyomatous lung tumors in women with uterine leiomyomas (so-called benign metastasizing leiomyomas). A report on 5 cases. Pathol Res Pract 192(3): 215-223.

8. Kayser K, Zink S, Schneider T, Dienemann H (2000) Benign metastasizing leiomyoma of the uterus: documentation of clinical, immunohistochemical and lectin-histochemical data of ten cases. Virchows Arch 437(3): 284-292.

9. Yoshitomi A, Nihon Kyobu Shikkan, Gakkai Zasshi (1994) A case of so called benign metastasizing leiomyoma 32(4): 373-377.

10. Horiuchi K, Yabe H, Mukai M, Morioka H, Udagawa Y, et al. (1998) Multiple smooth muscle tumors arising in deep soft tissue of lower limbs with uterine leiomyomas. Am J Surg Pathol 22(7): 897-901.

11. Groeneveld AB, Bosma A, Ceelen TL, Kouwenhoven TJ, Meuwissen SG, et al. (1986) Progressive and fatal course of a patient with a multifocal leiomyomatous tumor. Am J Gastroenterol 81(8): 702-707.

12. Cho KR, JD Woodruff, JI Epstein (1989) Leiomyoma of the uterus with multiple extrauterine smooth muscle tumors: a case report suggesting multifocal origin. Hum Pathol 20(1): 80-83.

13. Dürck H (1907) Ueber ein kontinvierlich durch die learned Hohlvene in das Herz vorwachsendes Fibromyom des Uterus. München Med Wochenschr 54: 1154.

14. Mandelbaum I, Pauletto FJ, Nasser WK (1974) Resection of a leiomyoma of the inferior vena cava that produced tricuspid valvular obstruction. J Thorac Cardiovasc Surg 67: 561-567.

15. Li B, Chen X, Chu Y-D, Li R-Y, Li W-D, et al. (2013) Intracardiac leiomyomatosis: a comprehensive analysis of 194 cases. Interactive Cardiovascular and Thoracic Surgery 17(1): 132-138.

16. Galandiuk S, Fazio VW, Jagelman DG, Lavery IC, Weakley FA, et al. (1987) Villous and tubulovillous adenomas of the colon and rectum. A retrospective review 1964-1985. Am J Surg 153(1): 41-47.

17. Allen B, C Rader, A Babigian (2007) Giant lipomas of the upper extremity. Can J Plast Surg 15(3): 141-144.
18. Bell SW, RL Kempson, MR Hendrickson (1994) Problematic uterine smooth muscle neoplasms. A clinicopathologic study of 213 cases. Am J Surg Pathol 18(6): 535-558.

19. Kjerulff KH, Langenberg P, Seidman JD, Stolley PD, Guzinski GM, et al. (1996) Uterine leiomyomas. Racial differences in severity, symptoms and age at diagnosis. J Reprod Med 41(7): 483-490.

20. Prayson RA, WR Hart (1995) Pathologic considerations of uterine smooth muscle tumors. Obstet Gynecol Clin North Am 22(4): 637-657.

21. Robboy SJ, Bentley RC, Butnor K, Anderson MC, et al. (2000) Pathology and pathophysiology of uterine smooth-muscle tumors. Environ Health Perspect 108(Suppl 5): 779-784.

22. Tietze L, Günther K, Hörbe A, Pawlik C, Klosterhalfen B, et al. (2000) Benign metastasizing leiomyoma: a cytogenetically balanced but clonal disease. Hum Pathol 31(1): 126-128.

23. El Rifai W, Maarit Sarlomo Rikala, Sakari Knuutila, Markku Miettinen, et al. (1998) DNA copy number changes in development and progression in leiomyosarcomas of soft tissues. Am J Pathol 153(3): 985-990.

24. Canzonieri V, D Amore ES, Bartoloni G, Piazza M, Blandamura S, et al. (1994) Leiomyomatosis with vascular invasion. A unified pathogenesis regarding leiomyoma with vascular microinvasion, benign metastasizing leiomyoma and intravenous leiomyomatosis. Virchows Archiv 425(5): 541-545.

25. Kempson RL, MR Hendrickson (1988) Pure mesenchymal neoplasms of the uterine corpus: selected problems. Semin Diagn Pathol 5(2): 172198.

26. Pocock E, JR Craig, WK Bullock (1976) Metastatic uterine leiomyomata. A case report Cancer 38(5): 2096-2100.

27. Lek M, Karczewski KJ, Minikel EV, Samocha KE, Banks E, et al. (2016) Analysis of protein-coding genetic variation in 60,706 humans. Nature 536(7616): 285-291.

28. Dong C, Wei P, Jian X, Gibbs R, Boerwinkle E, et al. (2015) Comparison and integration of deleteriousness prediction methods for nonsynonymous SNVs in whole exome sequencing studies. Hum Mol Genet 24(8): 21252137.

29. Liu X, X Jian, E Boerwinkle (2011) dbNSFP: a lightweight database of human nonsynonymous SNPs and their functional predictions. Hum Mutat 32(8): 894-899.

30. Liu X, X Jian, E Boerwinkle (2013) dbNSFP v2.0: a database of human non-synonymous SNVs and their functional predictions and annotations. Hum Mutat 34(9): E2393-2402.

31. Liu X, Wu C, Li C, Boerwinkle E (2016) dbNSFP v3.0: A One-Stop Database of Functional Predictions and Annotations for Human Nonsynonymous and Splice-Site SNVs. Hum Mutat 37(3): 235-241.

32. Hartmaier (2017) High-throughput genomic profiling of adult solid tumors reveals novel insights into cancer pathogenesis. Cancer Res 77(9): 2464-2475.

33. Walker F (2009) Growth factor receptor expression in anal squamous lesions: modifications associated with oncogenic human papillomavirus and human immunodeficiency virus. Hum Pathol 40(11): 1517-1527.

34. Fogel BL (2011) Interpretation of genetic testing: variants of unknown significance. Continuum (Minneap Minn) 17(2 Neurogenetics): 347-352.

35. Rivera JA (2004) Hormonal manipulation of benign metastasizing leiomyomas: report of two cases and review of the literature. J Clin Endocrinol Metab 89(7): 3183-3188.

36. Arai T (2000) Natural decrease of benign metastasizing leiomyoma. Chest 117(3): 921-922.

37. Chan CY (2004) A woman with a pelvic mass and pulmonary nodules. Br J Radiol 77(917): 459-460.

38. BannerAS(1981)Efficacyofoophorectomyinlymphangioleiomyomatosis and benign metastasizing leiomyoma. N Engl J Med 305(4): 204-209. 
39. Evans AJ (1986) Metastasizing leiomyoma of the uterus and hormonal manipulations. Case report. Br J Obstet Gynaecol 93(6): 646-648.

40. Saynajakangas O, AG Maiche, KA Liakka (2004) Multiple progressive pulmonary leiomyomatous metastases treated with tamoxifen-a case report with a review of the literature. Acta Oncol 43(1): 113-114.

41. Jacobson TZ, EJ Rainey, CW Turton (1995) Pulmonary benign metastasising leiomyoma: response to treatment with goserelin. Thorax 50(11): 1225-1226.

42. Glasier AF (2002) Administration of an antiprogesterone up-regulates estrogen receptors in the endometrium of women using Norplant: a pilot study. Fertil Steril 77(2): 366-372.

43. Bulun SE, ER Simpson, RA Word (1994) Expression of the CYP19 gene and its product aromatase cytochrome P450 in human uterine leiomyoma tissues and cells in culture. J Clin Endocrinol Metab 78(3): 736-743.

44. Shozu M (2003) Successful treatment of a symptomatic uterine leiomyoma in a perimenopausal woman with a nonsteroidal aromatase inhibitor. Fertil Steril 79(3): 628-631.
45. Sumitani H (2000) In situ estrogen synthesized by aromatase P450 in uterine leiomyoma cells promotes cell growth probably via an autocrine/ intracrine mechanism. Endocrinology 141(10): 3852-3861.

46. Walker CL (2000) Preclinical evidence for therapeutic efficacy of selective estrogen receptor modulators for uterine leiomyoma. J Soc Gynecol Investig 7(4): 249-256.

47. Rao UN, SD Finkelstein, MW Jones (1999) Comparative immunohistochemical and molecular analysis of uterine and extrauterine leiomyosarcomas. Mod Pathol 12(11): 1001-1009.

48. Maredia R (1998) Benign metastasizing leiomyoma in the lung. Radiographics 18(3): 779-782.

49. Andrade LA (1998) Intravenous leiomyomatosis of the uterus. A report of three cases. Pathol Oncol Res 4(1): 44-47.

50. Kocica MJ (2005) Intravenous leiomyomatosis with extension to the heart: rare or underestimated? J Thorac Cardiovasc Surg 130(6): 17241726.

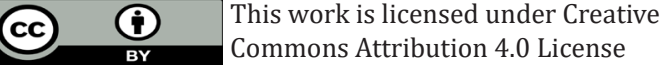

To Submit Your Article Click Here:

Submit Article

DOI: 10.32474/OAJOM.2018.02.000137

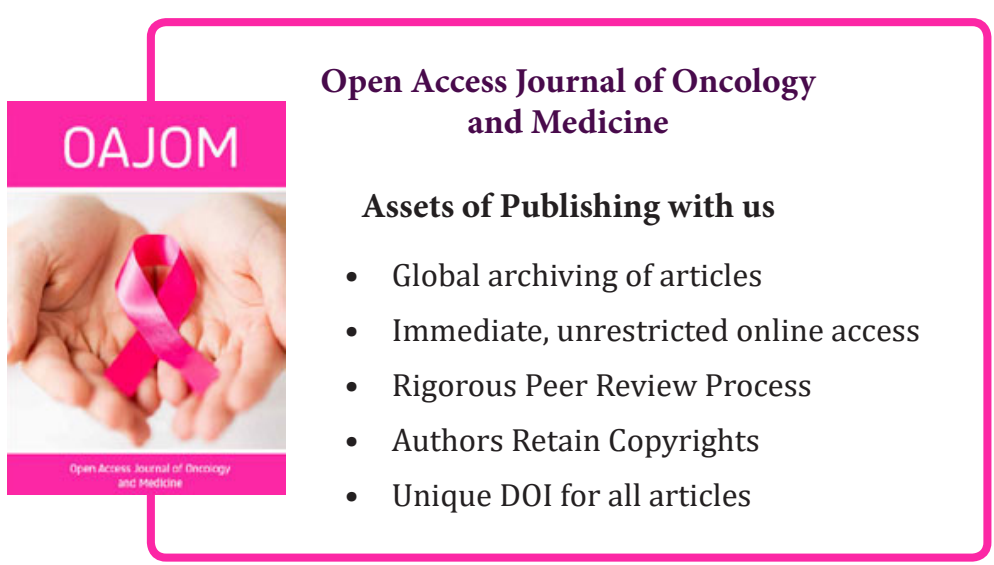

